\title{
sciendo
}

\author{
ANGELIKA KUCZYŃSKA, ŁUKASZ KWIETNIEWSKI, WIKTOR KUPISZ, \\ JOANNA KRUK-BACHONKO, WITOLD KRUPSKI
}

\section{Digital breast tomosynthesis (DBT) value in breast mass detection}

\begin{abstract}
Epidemiologically, breast cancer is the most common cancer in middle-aged women and it is one of the leading causes of cancer-related deaths. Middle-aged patients are covered by screening tests - digital mammography, often supplemented with ultrasound (US) breast examination. Other radiological tests in the diagnosis of breast cancer include such techniques as tomosynthesis, spectral mammography and magnetic resonance imaging (MRI). Many research groups around the world have demonstrated superiority of tomosynthesis in detecting focal lesions in breasts when compared to conventional mammography. Tomosynthesis usage was proposed for screening studies as a test of choice and for radiologically-guided tissue biopsies of suspicious tissue lesions.
\end{abstract}

Keywords: digital breast tomosynthesis, breast cancer, mammography.

DOI: $10.2478 /$ pjph-2020-0001

\section{INTRODUCTION}

Breast cancer is the most common neoplasm in population of Polish women and it is the most common cause of death in women between 40 and 55 years of age. Statistical data from Polish women population indicate that breast cancer morbidity rate in 2017 counted 18,529 cases and mortality rate counted 6670 cases. Effective treatment and reduction of mortality rate in the group of patients with breast cancer is possible mainly due to early diagnosis of the cancer at its initial stage.

The basic physical examination in women of all ages is regular self-examination in the form of breast palpation in standing and lying position. Imaging diagnostics tests are necessary for an extension of the diagnostics in case of palpation-detectable changes of breast tissue or observed changes in the skin of the breast, like skin pulling, orange peel and redness of the skin.

The diagnostic examination of choice in mature women with fat tissue predominance in breasts is full-field digital mammography (FFDM), while in young women with glandular tissue predominance it is breast ultrasound examination. In some cases, when the result of diagnostic test is unclear, a radiologist can suggest an additional radiological examinations such as digital breast tomosynthesis, spectral mammography and MRI of breasts [1-3]. Full-field digital mammography is a good tool for screening examinations in mature women. In Poland, population of women between 50 and 69 years old is included in screening mammographic examination once every two years. The adequate diagnostic method guarantees better and earlier detection of possible malignant lesions in breast and reduces mortality caused by breast cancer. Over $22 \%$ of malignant masses in fat rich breast cannot be detectable using FFDM and over $52 \%$ of the changes is undetectable in breast composed of high percentage of glandular tissue [4]. Mammography achieves the highest sensitivity in breast showing predominance of fat tissue and thus FFDM is dedicated for these cases. Pathological tissue masses and healthy glandular tissue have similar X-ray absorption degree and detection of malignant masses in glandular-tissue rich breast is difficult or even impossible. Other disadvantage of mammography is imposition of anatomical structures within whole breast structure and obscure suspicious masses. For such cases, DBT can be more precise tool for screening test or may serve as an alternative diagnostic tool for FFDM.

\section{AIM}

The aim of the study is to present possible advantages and disadvantages of tomosynthesis technique usage for medical diagnostic of breast based on our own diagnostic experience and review of available literature.

\section{MATERIALS AND METHODS}

Digital breast tomosynthesis (DBT) is executed using series of low-dose X-rays exposures by X-ray tube that moves around the breast in a line of prearranged arc. During the examination, breast is compressed by a compression paddle. According to data reported by some researches, good quality diagnostic examination is acquired with reduced compression of the breasts also [5]. Two examination techniques using DBT can be distinguished; the technique with continuous X-ray tube movement (continuous scanning mode with short pulses of radiation, movement artifacts can be detectable), and step and shoot tube motion (X-ray tube stops in different angles above breast 
and the X-rays are executed in particular positions and then the examination is bereft of movement artifacts). Complete set of X-ray projections images are reconstructed into thin image slices spaced at the distance of 0.5-1.0 mm.

The larger the angle used for the diagnostic examination, the more projections are obtained. At a $50^{\circ}$ angle, approximately 25 projections are created, and higher amount of projections contributes to the generation of more detailed $3 \mathrm{D}$ reconstructions and to higher detection of tissue malignant lesions and provided possibility to distinguish normal anatomical structures and structural tissues abnormalities.

\section{RESULTS}

Significant differences were proved in numerous reports and studies between full-field digital mammography and digital breast tomosynthesis in breast pathological mass and architectural distortion detection. Calcifications detection and shape estimation ability are comparable between mammography and digital breast tomosynthesis.

\section{DISCUSSION}

Digital breast tomosynthesis examination protocol is similar to routine mammography (Figure 1). As the result of DBT examination, two types of projections of each breast are achieved; the first craniocaudal (CC) and the second mediolateral oblique (MLO). Additionally, when the diagnosis based on both these projections is difficult, radiologist can extent basic examination protocol using other projections. Digital breast tomosynthesis examination protocol is performed with breast compression, the same as in case of FFDM. The compression of the whole breast can be performed or simply the part of the breast with suspicious mass can be compressed only.

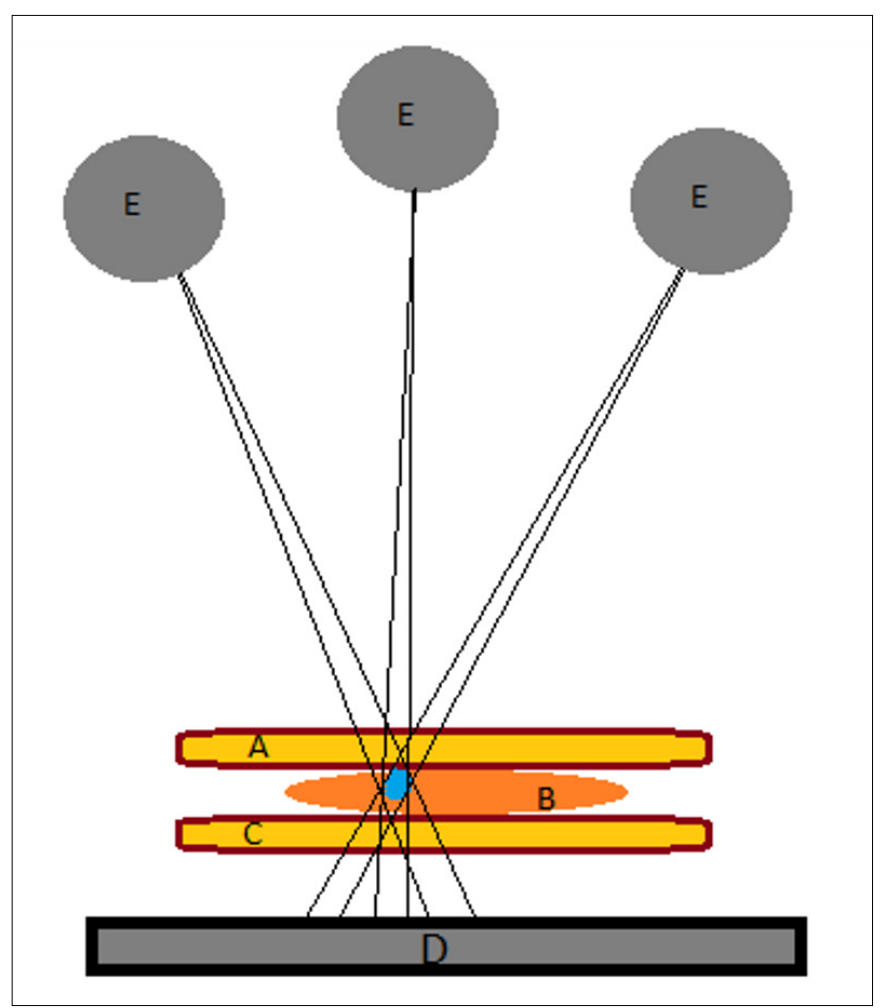

FIGURE 1. Scheme of the principles of diagnostic examination of breast using DBT technique. A - compression paddle; B - breast structure; C - breast support; D - X-ray detector; E - X-ray tube.
Main advantages of DBT technique in comparison with FFDM are high-contrast visibility, greater visibility of the margins of pathological masses and more precise determination of lesion size [6-10].

\section{Radiation dose}

According to ALARA (as low as reasonably achievable) scale, diagnostic examination should be performed using the lowest possible radiation dosage [8]. X-ray dosage in each image projection in DBT technique is lower than X-ray dosage resulting from FFDM examination. Digital breast tomosynthesis examination counts approximately 25 single projections in each breast. Although X-ray dosage in each projection in DBT examination is lower, combination of all projections of DBT examination causes patient radiation exposure increases twofold $[9,22]$. Radiation dosage for patient depends on quantity of projections and thickness of breast tissue. Average glandular dose (AGD) increases in accordance with increased breast tissue thickness. Average glandular dose is about 30-60\% greater in DBT examination than in case of FFDM. However, numerous researches prove that the diagnostic advantages of DBT are greater than the risk related to higher radiation dosage in patient, especially for patients with breast thickness equal to or lower than $63 \mathrm{~mm}$ after compression $[3,11,23]$.

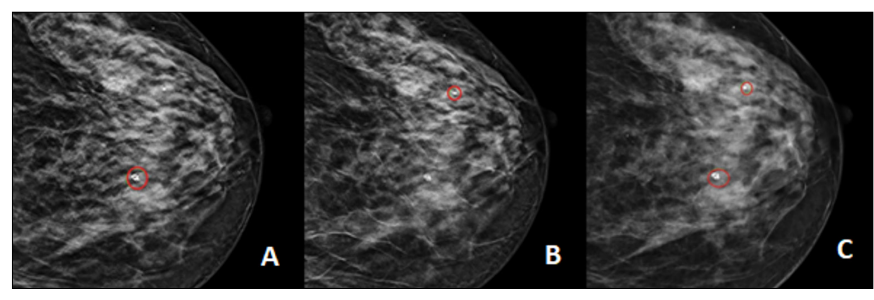

FIGURE 2. Craniocaudal (CC) projections of the left breast using digital breast tomosynthesis (A and $B$ ) and full-field digital mammography (C). Changes surrounded using red line circles show benign "popcorn" calcifications pattern (A), benign microcalcifications (B) as well as benign "popcorn" calcifications pattern in medial quadrant and benign microcalcifications in lateral quadrant $(C)$.

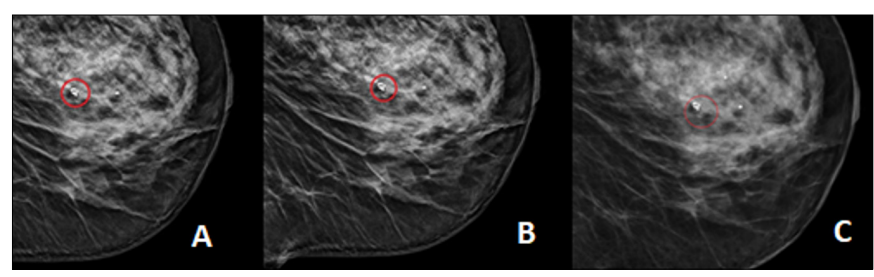

FIGURE 3. Mediolateral oblique (MLO) projections of the left breast using digital breast tomosynthesis ( $A$ and $B$ ) and full-field digital mammography (C). Changes surrounded using red line circles show benign "popcorn" calcifications pattern $(\mathrm{A}, \mathrm{B}, \mathrm{C})$. 
TABLE 1. Typically benign calcifications.

\begin{tabular}{|c|c|}
\hline $\begin{array}{c}\text { Typically benign } \\
\text { calcifications }\end{array}$ & morphology \\
\hline Vascular & Railways pattern \\
\hline Postinflammatory & $\begin{array}{l}\text { Round, oval, } \\
\text { "popcorn" type }\end{array}$ \\
\hline In cysts' walls & $\begin{array}{l}\text { Circinate, } \\
\text { round, oval }\end{array}$ \\
\hline Milk of calcium & $\begin{array}{c}\text { "Tea cup", } \\
\text { "crescent shaped" }\end{array}$ \\
\hline In benign dysplasia & $\begin{array}{l}\text { Punctate, small, } \\
\text { difficult to dif- } \\
\text { ferentiate with } \\
\text { malignant calcifi- } \\
\text { cations }\end{array}$ \\
\hline
\end{tabular}

\section{Calcification}

The assessment of the nature of calcifications in the mammary gland is very important. Some of the calcifications are typically benign (round or oval), others (linear, branching) can give high suspicion of possible malignant neoplastic process (Figure 1,2). Some of the studies show that digital mammography has predominance for estimation of shape of calcifications in comparison with DBT [12]. However, calcifications are also easily visible in examination using DBT technique. In some cases, visibility of microcalcifications can be limited by artifacts resulting from X-ray tube movement and estimation of shape of calcifications in DBT and FFDM $[13,14]$. The best visibility of microcalcifications can be achieved with the use of slab technique and then employing maximum intensity projection (MIP) post-processing technique, method that visualize voxels with highest Hounsfield number. Maximum intensity projection technique shows all the calcifications in a volume.

In many cases, the assessment of calcifications using DBT technique is comparable to routine mammography, and although sometimes DBT may show a loss of a characteristic image and morphology of calcifications, a single DBT image can obtain a microcalcification image without obscuring structures which can facilitate both their identification and determination of morphology.

Tables 1 and 2 present various characteristics of calcifications. Typically benign calcifications are round, oval, "popcorn" pattern, circinate, "railways" pattern (Table 1). Calcifications with suspicious morphology are visible as branching, linear, in size less than $0.5 \mathrm{~mm}$, different density (Table 2). Estimation of calcifications is often comparable in DBT and FFD [12-14].
TABLE 2. Calcifications with suspicious morphology.

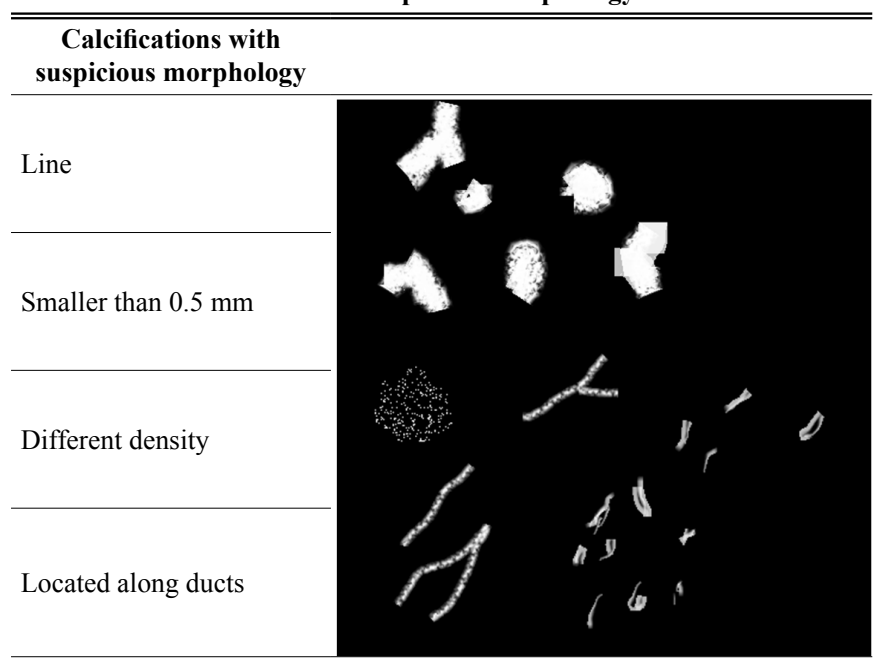

\section{Pathological tissue mass types}

According to the BI-RADS classification, breast masses are recognized as opacities seen in two orthogonal views. These can be both with suspicious morphology or typically benign. Radiologists may describe the characteristics of pathological mass lesions in terms of shape, texture and appearance of surrounding region. There are scientific evidences that irregular, microlubulated and poorly defined masses have greater likelihood of neoplastic malignancy. In FFDM imaging technique, tissue masses surrounded with dense glandular tissue are usually very difficult to detect or even undetectable. DBT technique shows the advantage that eliminates artifacts from normally dense glandular tissue and results in better detection of pathological masses, even masses with the size about 7-8 mm. In long-term trials, DBT technique shows superior value for size changes observation between following examination also [12].

Benefits resulting from diagnostic usage of tomosynthesis for the detection of focal lesions include increased visibility of pathological breast masses and detection of masses smaller than $8 \mathrm{~mm}$. Digital breast tomosynthesis can also detect spiculated masses with about $7 \mathrm{~mm}$ diameter, and the spiculated tissue masses are better visible even at early stages of their development [12].

\section{Architectural distortion (AD)}

Digital breast tomosynthesis improves visibility of architectural distortion and reduces pseudo-AD effect, when compared to FFDM. Long-term trials show superiority of DBT in AD detection in comparison with FFDM, breast US and Breast MRI [2]. It was proposed to increase diagnostic usage of DBT to assess architectural distortion in breasts [12].

Benefits resulting from diagnostic usage of tomosynthesis in the diagnosis of architectural distortion include reduction in false positive results of $\mathrm{AD}$ diagnosis and an increase in proper AD diagnosis.

Numerous previous reports have confirmed that using DBT causes the increase of the detection efficiency of malignant lesions in the mammary glands, and the decrease in the number of false negative results and the reduction of repeated calls of patients for supplementary tests with false positive results [6-9]. Digital breast tomosynthesis is more sensitive than fullfield digital mammography in detecting focal lesions in the breast. The sensitivity of tomosynthesis ranges from $60 \%$ 
to $100 \%$ and the specificity of the detected changes varies from $54 \%$ to $100 \%$ [15]. The greatest advantages of 3D mammography with tomosynthesis compared to conventional $2 \mathrm{D}$ mammography is greater efficiency in the detection of microcalcifications $[13,14]$, elimination of overlapping of many structures, better assessment of the outline of the lesion and the extent of the tumor process, and more precise assessment of the number of pathological lesions [6-10]. Given the benefits of tomosynthesis and slightly higher exposure to ionizing radiation, when compared to conventional mammography, tomosynthesis may be considered as an alternative with greater diagnostic value, and it is recommended not only as an extension of medical diagnostics but also as a basic examination $[9,16,17]$. An important issue in the application of DBT is the increase in the detection of breast cancer in the early stages of its development and, as a result, significantly contributes to the reduction of premature mortality in the female population due to cancer [18-20].

\section{CONCLUSIONS}

The numerous reports and studies show that the main advantages of DBT are earlier establishment of proper diagnosis and lower rate of unnecessary recall for verification of the suspicious changes [21]. Digital breast tomosynthesis, as first choice method of radiological breast examination, can result in reduction of repeated examinations using FFDM screening tests. Considering that some pathological changes are visible only in DBT examination, the diagnostic usage of this technique enables performance of the radiologically-guided breast mass biopsy in patients.

\section{REFERENCES}

1. Rogaliński M. Tomosynteza - nowa nadzieja mammografii. IFM. 2014;3:91-2.

2. Roganovic D, Djilas D, Vujnovic S, et al. Breast MRI, digital mammography and breast tomosynthesis: Comparison of three methods for early detection of breast cancer. Bosn J Basic Med Sci. 2015;15(4):64-8.

3. Kisielewicz K, Dziecichowicz A, Sapikowska A, et al. Dawki dla pacjentek poddawanych cyfrowej tomosyntezie w mammografii. IFM. 2019;8:267.

4. Tabár L, Vitak B, Chen TH-H, et al. Swedish two-county trial: impact of mammographic screening on breast cancer mortality during 3 decades. Radiology. 2011;260(3):658-63.

5. Agasthya GA, D'Orsi E, Kim YJ, et al. Can breast compression be reduced in digital mammography and breast tomosynthesis? AJR. 2017;209(5):32232.

6. Gilbert FJ. Digital breast tomosynthesis (DBT): a review of the evidence for use as a screening tool. Clin Radiol. 2016;71(2):141-50.

7. Skaane P. Breast cancer screening with digital breast tomosynthesis. Breast Cancer. 2017;24(1):32-41.

8. Nguyen T, Levy G, Poncelet E, et al. Overview of digital breast tomosynthesis: Clinical cases, benefits and disadvantages. Diagn Interv Imaging. 2015;96:843-59.

9. Hooley RJ, Durand MA, Philpotts LE. Advances in digital breast tomosynthesis. AJR. 2017;208:256-66.

10. Johnson MM. Full-field digital mammography and digital breast tomosynthesis. Radiol Technol. 2017;88(3):299M-319M.

11. Spak DA, Plaxco JS, Santiago L, et al. BI-RADS ${ }$ fifth edition: A summary of changes. Diagn Interv Imaging. 2017;98(3):179-90.

12. Mall S, Lewis S, Brennan P, Noakes J. The role of digital breast tomosynthesis in the breast assessment clinic: a review. J Med Radiat Sci. 64 2017;203-11.

13. Kopans D, Gavenonis S, Halpern E, Moore R. Calcifications in the breast and digital breast tomosynthesis. Breast J. 2011;17:638-44.

14. Spangler ML, Zuley ML, Sumkin JH, et al. Detection and classification of calcifications on digital breast tomosynthesis and 2D digital mammography: A comparison. Am J Roentgenol 2011;196:320-4.

15. Garcia-Leon FJ, Liamos-Mendez A, Jsabel-Gómez R. Digital tomosynthesis in breast cancer: A systematic review. Radiologia. 2015; 57(4):333-43.

16. Tabár L, Yen AMF, Wu WYY, et al. Insights from the breast cancer screening trials: How creening affects the natural history of breast cancer and implications for evaluating service screening programs. Breast $\mathrm{J}$. 2015;21:13-20.

17. Funaro K, Drukteinis J, Falcon S. Screening mammography and digital breast tomosynthesis: Controversies. South Med J. 2017;110(10):607-13.

18. Mandelson MT, Oestreicher N, Porter PL, et al. Breast density as a predictor of mammographic detection: comparison of interval-and screen-detected cancers. J Natl Cancer Inst. 2000;92:1081-7.

19. Kolb TM, Lichy J, Newhouse JH. Comparison of the performance of screening mammography, hysical examination, and breast US and evaluation of factors that influence them: an analysis of 27,825 patient evaluations. Radiology. 2002;225:165-75

20. Pisano ED, Gatsonis C, Hendrick E, et al. Diagnostic performance of digital versus film ammography for breast-cancer screening. N Engl J Med. 2005; 353:1773-83.

21. Smith RA, Duffy SW, Gabe R, et al. The randomized trials of breast cancer screening: what have we learned? Radiol Clin North Am. 2004;42:793806

22. Svahn TM, Houssami N, Sechopoulos I, et al. Review of radiation dose estimates in digital breast tomosynthesis relative to those in two-view fullfield digital mammography. Breast 2015;24:93-9.

23. Shin SU, Chang JM, Bae MS, et al. Comparative evaluation of average glandular dose and breast cancer detection between single-view digital breast tomosynthesis (DBT) plus single-view digital mammography (DM) and two-view DM: correlation with breast thickness and density. Eur Radiol 2015; 25:1-8.

Corresponding author

Angelika Kuczyńska

II Department of Medical Radiology, Medical University of Lublin

16 Staszica St., Lublin 20-081, Poland.

tel: +48815321084

E-mail: angelikakuczynska@umlub.pl 